\title{
Elimination du chrome par du charbon actif élaboré et caractérisé à partir de la coque du noyau de Balanites Aegyptiaca
}

\author{
Siragi D. B. MAAZOU, Halidou I. HIMA, Maman Mousbahou MALAM ALMA, \\ Zanguina ADAMOU et Ibrahim NATATOU*
}

Laboratoire Matériaux-Eau-Environnement (LAMEE), Faculté des Sciences et Techniques, Université Abdou Moumouni (UAM) de Niamey, BP 10662 Niamey, Niger.

*Auteur correspondant: E-mail : inatatou@yahoo.com

\section{RESUME}

Le charbon actif élaboré à partir de biomasses lignocellulosiques est une bonne voie de valorisation de déchets, vue ses applications. Ce travail porte sur l'élaboration du charbon actif à partir de Balanites aegyptiaca par activation chimique à l'acide ortho phosphorique, son application pour éliminer le chrome VI en solution. La concentration de l'acide, le temps d'imprégnation, la température de pyrolyse et le palier d'isotherme ont été variés. La caractérisation a porté sur la détermination des indices d'iode et de bleu de méthylène, des fonctions de surface et de $\mathrm{pH}$ au point de charge nulle $\left(\mathrm{pH}_{\mathrm{PCN}}\right)$. Le temps de contact, le $\mathrm{pH}$ du milieu, la masse de l'adsorbant et la concentration initiale de la solution ont été variés pour l'élimination du chrome. Les meilleurs indices d'iode et de bleu de méthylène obtenus sont : 989,86 mg/g et $613,17 \mathrm{mg} / \mathrm{g}$ respectivement. Les fonctions à la surface des charbons actifs sont de nature acide et leur $\mathrm{pH}_{\mathrm{PCN}}$ inférieur à 7 . Le rendement d'élimination du chrome varie de $81,64 \%$ à $98,77 \%$ dans les conditions : concentration initiale (70 $\mathrm{mg} / \mathrm{L}), \mathrm{pH}=2$, masse $(80 \mathrm{mg})$ et temps $(2 \mathrm{~h})$. A titre de comparaison, le charbon actif commercial de marque KGA a donné un taux de rabattement en chrome de $91,49 \%$.

(C) 2017 International Formulae Group. All rights reserved.

Mots clés : Biomasse, activation chimique, charbon actif.

\section{Elimination of the chromium by the elaborate activated coal and characterized from the c-ockle of the core of Balanites Aegyptiaca}

\begin{abstract}
Activated coal elaborated from biomasses lignocellulosiques is a good way of valorization of garbage, seen his applications. This work is about the development of the activated coal from Balanites aegyptiaca by chemical activation to ortho phosphoric acid, his application to eliminate the chromium VI in solution. The concentration of acid, the time of impregnation, the temperature of pyrolysis and the landing of isotherm have been varied. The characterization was about the determination of iodine index, methylene blue index, functions of surface and $\mathrm{pH}$ to point of zero charge $\left(\mathrm{pH}_{\mathrm{PZC}}\right)$. The time of contact, the $\mathrm{pH}$ of the middle, the mass of adsorbent and initial concentration of solution have been varied for the elimination of chromium. The best iodine index and methylene blue index gotten are: $989,86 \mathrm{mg} / \mathrm{g}$ and $613,17 \mathrm{mg} / \mathrm{g}$ respectively. The functions to the surface of activated coals are of acid nature and their $\mathrm{pH}_{\mathrm{PZC}}$ lower to 7 . The chromium elimination output
\end{abstract}


varies from $81,64 \%$ to $98,77 \%$ in the conditions: initial concentration $(70 \mathrm{mg} / \mathrm{L}), \mathrm{pH}=2$, mass $(80 \mathrm{mg})$ and time $(2 \mathrm{hs})$. To title of comparison, the commercial activated coal of KGA mark gave a rate of folding in chromium of $91,49 \%$.

(C) 2017 International Formulae Group. All rights reserved.

Keywords: Biomass, chemical activation, activated coal.

\section{INTRODUCTION}

Avec le développement socioéconomique, la production de déchets devient de plus en plus croissante dans le monde. En effet, la gestion de ces déchets reste un des principaux problèmes dans les pays en développement (PED). La quantité de déchets municipaux est en constante augmentation en raison de l'explosion démographique et de l'urbanisation (Clément et al., 2015). Parmi ces déchets, ceux qui sont biodégradables sont transformés par la nature. Par contre, ceux qui ne le sont pas ou le sont difficilement, s'accumulent dans la nature et la polluent.

Elaborer des charbons actifs à partir des déchets végétaux est intéressant du point de vue économique car c'est à partir des transformations simples qu'est effectuée une application directe de ces matériaux (Elena, 2002). L'industrie agro-alimentaire génère de grandes quantités de déchets dont la valorisation pour produire de l'énergie ou obtenir des matériaux est une voie prometteuse pour rendre les filières de production plus rentables (Rabilou, 2015). C'est dans ce cadre que des études sur ces précieux matériaux sont en cours un peu partout sur le continent et à travers le monde. Nous pouvons citer entre autres, les travaux de recherche menés sur : les coques de noix de coco (Drissa et al., 2009 ; Urbain et al., 2015 ; Clément et al., 2015), la coque d'arachide, les noix de coco et bois d'eucalyptus (Mbaye, 2014), les tourteaux de karité et tourteaux de coton (Ibrahim et al., 2012).

Notre contribution dans ce domaine à travers ce travail est d'élaborer des charbons actifs par activation chimique à partir de la coque des noyaux de Balanites Aegyptiaca avec l'acide ortho phosphorique comme agent activant. Ces noyaux sont des déchets agroalimentaires. L'utilisation de ces matériaux présente un double objectif, celui de fabriquer des charbons actifs de qualité à faible coût à partir de matériaux locaux et ainsi faisant donner à ces mêmes matériaux une valeur ajoutée (Ousmaila et al., 2016).

De nombreux scientifiques des différentes disciplines s'intéressent, de plus en plus, à l'identification et à l'élimination des polluants de l'environnement qui sont à la base de la morbidité et mortalité chez l'homme ou chez les animaux. A cet effet, on fait recourt souvent à l'adsorption sur charbon actif, notamment pour l'élimination des toxiques, des micropolluants organiques ou inorganiques de l'eau, la décoloration des huiles végétales et la purification de nombreux produits (Anatole et al., 2012). C'est dans cette optique, que nous allons traiter une solution de chrome VI avec les charbons actifs élaborés.

\section{MATERIEL ET METHODES Collecte et prétraitement}

La biomasse (noyaux de Balanites Aegyptiaca) a été collectée au niveau d'un dépotoir du marché KATAKO (NiameyNiger). Ces noyaux ont été lavés abondamment à l'eau puis séchés au soleil pendant 5 jours. Ils ont été concassés à l'aide d'un concasseur à mâchoire rotatif pour retenir la coque. Cette dernière a été broyée à l'aide d'un broyeur à boulets et ensuite tamisée grâce à deux tamis pour ne retenir que les particules de granulométrie comprise entre 1 et $1,6 \mathrm{~mm}$. Pour l'étape d'élaboration, la biomasse (particule de diamètre compris entre 1 et $1,6 \mathrm{~mm}$ ) a été mise à l'étuve à $105^{\circ} \mathrm{C}$ pendant $24 \mathrm{~h}$. Les noix de balanites aegyptiaca sous quelques formes sont présentées à la Figure 1. 


\section{Analyse de la matière première}

L'analyse de la matière première a porté sur l'analyse immédiate de la biomasse. Elle a consisté à déterminer le taux d'humidité, de cendres, les matières volatiles et le carbone fixe. Pour ce travail, l'analyse immédiate a été effectuée en suivant les normes AFNOR XP CEN/TS 14775 (cendres), AFNOR XP CEN/TS 14774-3 (humidité) et XP CEN/TS 15148 (matières volatiles).

\section{Taux d'humidité}

Des creusets en porcelaine ont été portés au four à $1000{ }^{\circ} \mathrm{C}$ pendant $3 \mathrm{~h}$ puis refroidit dans un dessiccateur. Le poids $\mathrm{P}$ de ces creusets a été pesé à l'aide d'une balance de précision au 1/10000. Une masse d'environ $2 \mathrm{~g}$ de biomasse a été introduite et le poids $\mathrm{P}_{1}$ a été noté. Ensuite, ils ont été placés (creusets + biomasse) à l'étuve à $105{ }^{\circ} \mathrm{C}$ pendant $24 \mathrm{~h}$. Après refroidissement, le poids $\mathrm{P}_{2}$ était pesé. Le taux d'humidité est donné par l'équation 1 suivante :

$$
(\mathbf{H} \%)=\frac{\mathbf{P}_{1}-\mathbf{P}_{2}}{\mathbf{P}_{1}-\mathbf{P}} \times 100
$$

\section{Matières volatiles (perte au feu)}

Les matières volatiles ont été obtenues en portant les creusets refroidit du taux d'humidité au four à $1000{ }^{\circ} \mathrm{C}$ pendant $3 \mathrm{~h}$. Enfin, un poids $\mathrm{P}_{3}$ a été noté après refroidissement. L'équation 2 donne le taux de matières volatiles de la biomasse.

$$
\text { (P. F\%) }=\frac{\mathbf{P}_{2}-\mathbf{P}_{3}}{\mathbf{P}_{2}-\mathbf{P}}
$$

\section{Taux de cendres}

Le taux de cendres a été déterminé en pesant une masse $\mathrm{m}_{1}$ de creuset vide dans lequel $1 \mathrm{~g}$ de biomasse est introduit et est noté la masse $\mathrm{m}_{2}$. L'ensemble était porté au four à $800{ }^{\circ} \mathrm{C}$ jusqu'à l'obtention de cendres. Enfin, la masse $\mathrm{m}_{3}$ était notée après refroidissement. Le taux de cendres est donné par l'équation 3 :

$$
(\mathbf{C} \%)=\frac{\mathbf{m}_{3}-\mathbf{m}_{1}}{\mathbf{m}_{2}-\mathbf{m}_{1}}
$$

Ces paramètres ont été déterminés avec une biomasse de diamètre inférieur à $200 \mu \mathrm{m}$.

\section{Activation chimique \\ Des solutions d'acide ortho} phosphorique ont été préparées à 10, 20, 30, et
$40 \%$ en masse à partir d'une solution mère titrant à $85 \%$ en masse.

Le protocole expérimental est tiré de la thèse de Mbaye (2014) qui a nécessité une imprégnation et une pyrolyse.

\section{Imprégnation}

La biomasse prétraitée est mise en contact sous agitation magnétique avec chacune des solutions d'acide en raison de 16 $\mathrm{g}$ de biomasse pour $100 \mathrm{~mL}$ de solution. Un temps d'imprégnation (temps d'agitation) de $24 \mathrm{~h}$ est retenu, mais aussi, un temps de $12 \mathrm{~h}$ et un temps de $18 \mathrm{~h}$ ont été expérimentés.

Après agitation, les échantillons sont filtrés sous Büchner puis mis à l'étuve à 105 ${ }^{\circ} \mathrm{C}$ pendant $24 \mathrm{~h}$ et sont ensuite refroidis dans un dessiccateur pour attendre la carbonisation (pyrolyse).

\section{Pyrolyse}

L'imprégnât a été mis au four (four à moufle modèle Heraeus) dans un creuset en verre borosilicaté à une température de $450{ }^{\circ} \mathrm{C}$ avec une vitesse de chauffe de $7,5{ }^{\circ} \mathrm{C} / \mathrm{min}$ et un palier $1 \mathrm{~h} 30 \mathrm{~min}$. Les variations de température ont été 300,400 et $500{ }^{\circ} \mathrm{C}$. Celles de palier d'isotherme ont été de $1 \mathrm{~h}, 2 \mathrm{~h}$ et $3 \mathrm{~h}$ successivement. Avant d'être retirés du four, les échantillons ont été refroidit d'abord dans ce dernier, puis dans un dessiccateur.

Ensuite le lavage a été effectué avec abondement de l'eau distillée pour avoir un $\mathrm{pH}$ compris entre 6,5 et 7 puis séchage à l'étuve à $105{ }^{\circ} \mathrm{C}$ pour $24 \mathrm{~h}$.

Par la suite, l'échantillon a été représenté par l'agent activant $\left(\mathrm{H}_{3} \mathrm{PO}_{4}\right)$ et le paramètre énuméré en gras dans le Tableau 1.

\section{Caractérisation chimique Rendement de pyrolyse}

C'est une caractéristique quantitative importante du charbon actif qui traduit la perte en masse de la biomasse pendant l'élaboration. Le rendement de pyrolyse est le rapport entre la masse finale et la masse initiale.

$$
\text { Rendement }(\%)=\frac{\mathbf{m}_{\mathbf{f}}}{\mathbf{m}_{\mathrm{i}}} \times 100
$$

\section{Détermination de l'indice d'iode}

L'indice d'iode (en $\mathrm{mg} / \mathrm{g}$ ) est la quantité en milligramme d'iode adsorbé par gramme de 
charbon actif dans une solution d'iode dont la normalité est de $0,02 \mathrm{~N}$. Il évalue la capacité d'adsorption des molécules de taille très petite par le charbon actif. La procédure utilisée a été celle du Centre d'Etude de Déchet, qui est une adaptation de la méthode du CEFIC 1989 et de la norme AWWA B $600-78$ tirée des travaux de Mbaye (2009).

Dans un bécher de $100 \mathrm{~mL}, 0,2 \mathrm{~g}$ du Charbon Actif $(\mathrm{CA})$ préalablement séché à l'étuve pendant $24 \mathrm{~h}$ à $105{ }^{\circ} \mathrm{C}$ est mise en contact avec $20 \mathrm{~mL}$ de la solution d'iode à $0,02 \mathrm{~N}$. Le mélange est agité pendant 4 à 5 minutes puis filtré. Par la suite, on procède au dosage des $10 \mathrm{~mL}$ du filtrat par une solution de thiosulfate de sodium à $0,1 \mathrm{~N}$. L'amidon a été utilisé comme indicateur coloré.

Ainsi, à l'équivalence (décoloration), on note le volume de la solution de thiosulfate versé : $\mathrm{V}_{\text {thio }}$. L'équation 5 donne l'indice d'iode.

$$
\mathbf{Q}_{\mathbf{I} 2}=\frac{\left(\mathbf{C}_{\mathbf{0}}-\frac{\mathbf{C}_{\text {thio }} \mathrm{v}_{\text {thio }}}{2 \mathbf{V}_{\mathbf{I} 2}}\right) \mathbf{M}_{\mathbf{I} 2} \mathbf{V}_{\text {ads }}}{\mathbf{m}_{\text {ca }}}
$$

* $\mathrm{QI}_{2}$ : capacité d'adsorption d'I $\mathrm{I}_{2}$ ou indice $\mathrm{d}^{\prime} \mathrm{I}_{2}$;

- $\mathrm{C}_{0}$ : concentration initiale de la solution $\mathrm{d}^{\prime} \mathrm{I}_{2}$ (en $\mathrm{mol} / \mathrm{L}$ ) ;

* $\mathrm{C}_{\text {thio }}$ : la concentration de $\mathrm{Na}_{2} \mathrm{~S}_{2} \mathrm{O}_{3}$ (en $\mathrm{mol} / \mathrm{L})$;

* $\mathrm{V}_{\text {thio }}$ : volume de $\mathrm{Na}_{2} \mathrm{~S}_{2} \mathrm{O}_{3}$ à l'équivalence (en $\mathrm{mL})$;

* $\mathrm{V}_{\mathrm{I}_{2}}$ : le volume d'iode dosé (en $\mathrm{mL}$ );

* $\mathrm{M}_{\mathrm{I}_{2}}$ : la masse molaire de l' $\mathrm{I}_{2}$ (en $\left.\mathrm{g} / \mathrm{mol}\right)$;

* Vads : le volume d'adsorption (en mL);

* $\mathrm{m}_{\mathrm{ca}}$ : masse de charbon actif utilisée (en g).

Détermination de l'indice de bleu de méthylène

L'indice de bleu de méthylène $(\mathrm{BM})$ exprimé en $\mathrm{mg} \cdot \mathrm{g}^{-1}$ représente la capacité d'adsorption des molécules de taille moyenne en vue d'évaluer les mésopores et les macrospores. La procédure utilisée est celle de la méthode du Centre Européen des Fédérations de l'Industrie Chimique (CEFIC, 1989).

Dans un erlenmeyer de $250 \mathrm{~mL}, 0,1 \mathrm{~g}$ de charbon actif préalablement séché et 100 $\mathrm{mL}$ de solution de bleu de méthylène à $1,944.10^{-5} \mathrm{M}$ ont été introduit. Le mélange est agité pendant 20 minutes puis filtré. La concentration résiduelle en bleu de méthylène a été déterminée à l'aide d'un spectrophotomètre UV- visible (marque ZUZI, modèle 4101) à une longueur d'onde de $620 \mathrm{~nm}$. Ainsi, l'indice de bleu de méthylène a été donné par la relation 6 suivante :

$$
\mathbf{Q}_{\mathrm{BM}}=\frac{\left(\mathrm{C}_{\mathrm{i}}-\mathrm{C}_{\mathrm{r}}\right) \mathbf{V M}}{\mathbf{m}_{\mathrm{ca}}} \times \mathbf{1 0 0}
$$

- $\mathrm{Q}_{\mathrm{BM}}$ capacité d'adsorption du CA (en $\mathrm{mg} / \mathrm{g}$ ) ;

* Ci concentration initiale de la solution de $\mathrm{BM}$ (en mol/L) ;

* Cr concentration résiduelle de la solution de BM (en mol/L) ;

* V volume de la solution de BM (en mL) ;

* M masse molaire de BM ;

* $\mathrm{m}_{\mathrm{ca}}$ masse du CA utilisée (en g).

Détermination des fonctions de surface

La méthode adoptée pour la détermination des fonctions de surface a été celle de Boehm (1966) tirée des travaux de Ibrahim et al. (2012) qui est une méthode de dosage retour. Les groupements basiques sont dosés dans leur globalité alors que les groupements acides sont dosés séparément. Le protocole expérimental est le suivant.

Une masse de $0,2 \mathrm{~g}$ du charbon actif a été mis en contact avec $20 \mathrm{~mL}$ de chacune des solutions aqueuses de $\mathrm{NaOH}, \mathrm{Na}_{2} \mathrm{CO}_{3}$, $\mathrm{NaHCO}_{3}, \mathrm{C}_{2} \mathrm{H}_{5} \mathrm{ONa}$ et $\mathrm{HCl}$ à $0,1 \mathrm{M}$. Chaque solution a été agitée pendant $24 \mathrm{~h}$ afin de s'assurer qu'un maximum de groupement de surface de charbon actif a réagi. Par la suite, on procède à la filtration des différentes solutions. Ensuite, $10 \mathrm{~mL}$ du filtrat de chacune des solutions ont été dosés. Les solutions basiques sont dosées par l'acide chlorhydrique à $0,1 \mathrm{M}$ et la solution acide par de la soude à $0,1 \mathrm{M}$.

La fonction cherchée est donnée en milliéquivalent par gramme par la formule suivante :

$$
\text { néqR= NiVi }- \text { NfVf } \quad \text { (7) }
$$
ayant réagi ;

néqR le nombre d'équivalent gramme

NiVi le nombre d'équivalant gramme avant la réaction ; 
NfVf le nombre d'équivalant gramme après la réaction.

En effet, le $\mathrm{NaHCO}_{3}$ neutralise seulement les fonctions carboxyliques, le $\mathrm{Na}_{2} \mathrm{CO}_{3}$ neutralise les fonctions carboxyliques et les fonctions lactones, le $\mathrm{NaOH}$ dose les fonctions carboxyliques, lactones et phénols, et le $\mathrm{C}_{2} \mathrm{H}_{5} \mathrm{ONa}$ les fonctions carbonyles. Pour ce qui est des fonctions basiques, dans leur globalité elles sont neutralisées par la solution l'acide chlorhydrique.

\section{Détermination de $\mathrm{pH}$ au point de charge nulle $\left(\mathbf{p H}_{\mathrm{PCN}}\right)$}

Il existe un $\mathrm{pH}$ pour lequel le charbon actif est électriquement neutre en solution. C'est-à-dire que la somme des charges à la surface est nulle. $\mathrm{Ce} \mathrm{pH}$ est appelé $\mathrm{pH}$ au point de charge nulle $\left(\mathrm{pH}_{\mathrm{PCN}}\right)$.

Pour déterminer le $\mathrm{pH}_{\mathrm{PCN}}$, la méthode de la première bissectrice a été utilisée. Cette méthode consiste à préparer des solutions de chlorure de sodium $(\mathrm{NaCl}) 0,1 \mathrm{M}$ à des $\mathrm{pH}$ différents ; 2, 4, 6, 8, 10. Les valeurs des $\mathrm{pH}$ ont été ajustées avec un pH-mètre modèle $\mathrm{HI}$ 991001 en utilisant des solutions d'hydroxyde de sodium et d'acide chlorhydrique. Ainsi, 0,1 g de CA ont été mis en contact avec $20 \mathrm{~mL}$ de chaque solution par échantillon. Le mélange a été mis sous agitation magnétique pendant 72 h. La suspension a été filtrée à travers un papier filtre et le $\mathrm{pH}$ du filtrat mesuré avec un $\mathrm{pH}$ mètre.

Ainsi, on trace la courbe $\mathrm{pH}_{\mathrm{f}}=\mathrm{f}\left(\mathrm{pH}_{\mathrm{i}}\right)$. Le point d'intersection entre cette courbe et la première bissectrice donne le $\mathrm{pH}$ au point de charge nulle du charbon actif considéré.

\section{Elimination du chrome}

La solution de chrome traitée est celle de dichromate de potassium $\left(\mathrm{K}_{2} \mathrm{Cr}_{2} \mathrm{O}_{7}\right)$. En effet, l'adsorption du chrome VI sur le charbon actif est effectuée comme suit :

$>$ une masse $\mathrm{m}$ du $\mathrm{CA}$ a été pesée à l'aide d'une balance de précision au 1/1000 et était mise dans un bécher de $100 \mathrm{~mL}$;

- un volume de $50 \mathrm{~mL}$ de la solution de chrome de concentration connue a été mis dans le bécher et le mélange était porté sous agitation magnétique pour un temps bien définie; après l'agitation, le mélange a été filtré à travers un papier filtre sans cendre et la concentration résiduelle en chrome était mesurée à l'aide d'un MP-AES (MicroPlasma Atomic Emission Spectroscopy) de Argilent Technologies, modèle 4200.

La capacité d'adsorption du chrome est donnée par la relation suivante :

$$
\mathbf{Q}=\frac{\left(\mathbf{C}_{\mathbf{i}}-\mathbf{C}_{\mathbf{f}}\right) \mathbf{V}}{\mathbf{m}}
$$

* Q la capacité d'adsorption du chrome ;

* Ci la concentration initiale de la solution de chrome en $\mathrm{mg} / \mathrm{L}$;

* Cf la concentration finale de la solution de chrome en $\mathrm{mg} / \mathrm{L}$;

* V le volume de la solution de chrome (50 $\mathrm{mL}$ ) ;

* m la masse du charbon actif en g.

Pour avoir la meilleure condition d'élimination du chrome, les paramètres suivants ont été variés : le temps de contact, la masse du charbon actif, le $\mathrm{pH}$ de la solution et sa concentration initiale. Ces derniers ont été étudiés pour l'échantillon noté $\mathrm{H}_{3} \mathrm{PO}_{4}-12 \mathrm{~h}$ car il nous a donné d'indices d'iode et de bleu de méthylène les plus élevés.

Ainsi, on peut aussi déterminer le rendement d'extraction du chrome grâce à la relation suivante :

$$
\mathbf{r}(\%)=\frac{\left(\mathbf{C}_{\mathbf{i}}-\mathbf{C}_{\mathbf{f}}\right)}{\mathbf{C}_{\mathbf{i}}} \times \mathbf{1 0 0}
$$

\section{Effet de temps de contact}

La variation de temps de contact a été réalisée dans les conditions suivantes : $\mathrm{Ci}=50$ $\mathrm{mg} / \mathrm{L}, \mathrm{V}=50 \mathrm{~mL}$ et $\mathrm{m}=50 \mathrm{mg}$. Le mélange étant en agitation, des prélèvements ont été effectués à intervalle de temps pour déterminer la concentration restante en chrome. Le temps de contact maximum a été de 2 heures.

\section{Effet de la masse du charbon actif}

Pour l'augmentation de la surface de contact (variation de masse du charbon actif), le temps de contact a été de $2 \mathrm{~h}$. Cependant, les masses considérées ont été de 20, 40, 60 et $80 \mathrm{mg}$ de charbon actif pour $50 \mathrm{~mL}$ de solution de chrome à $70 \mathrm{mg} / \mathrm{L}$.

\section{Effet du pH de la solution du chrome}

La variation du $\mathrm{pH}$ de la solution de chrome est un paramètre essentiel pour l'adsorption car il existe quatre formes 
d'oxydes de chrome en fonction du $\mathrm{pH}$ (Mbaye, 2014). Pour cette partie, le temps de contact a été fixé à $2 \mathrm{~h}$, la masse en adsorbant à $80 \mathrm{mg}$ et la concentration de la solution à 70 $\mathrm{mg} / \mathrm{L}$. Ainsi, l'élimination du chrome a été faite à $\mathrm{pH}=2,4,6,8$ et 10.

Effet de la concentration initiale de la solution

Enfin, pour la variation de la concentration, les valeurs des paramètres ci- dessus pour lesquels l'adsorption est meilleure ont été conservées $(\mathrm{t}=2 \mathrm{~h}, \mathrm{~m}=80 \mathrm{mg}$ et $\mathrm{pH}=$ 2).

Cependant, la variation de concentration a été effectuée pour les valeurs suivantes : 40, 60, 80, 100 et $120 \mathrm{mg} / \mathrm{L}$ en chrome dans la solution.

Pour s'assurer de la reproductibilité du résultat, les analyses ont été répétées trois fois.

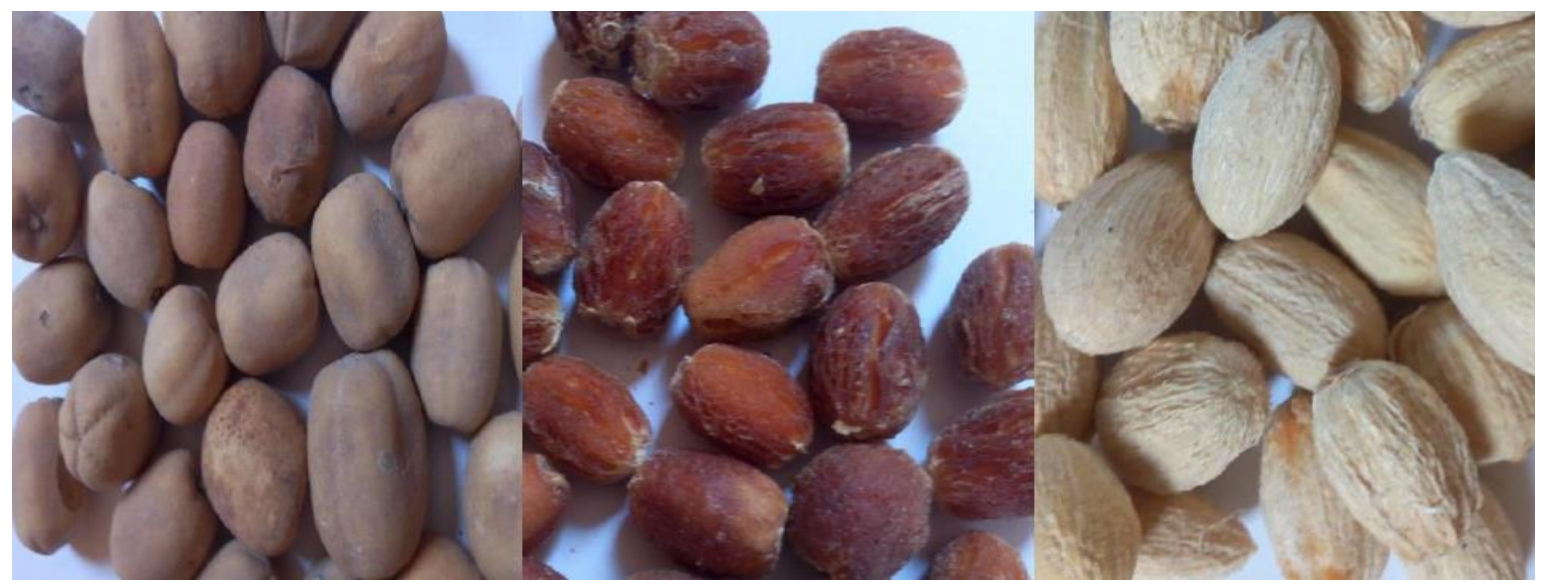

Figure 1 : Noix entière, noix sans l'enveloppe extérieure et noix nue de balanites aegyptiaca de gauche à droite respectivement.

Tableau 1 : Conditions d'activation.

\begin{tabular}{lcccc}
\hline $\begin{array}{c}\text { Concentration } \\
\text { en acide en \% }\end{array}$ & $\begin{array}{c}\text { Temps } \\
\text { d'imprégnation en } \mathbf{h}\end{array}$ & $\begin{array}{c}\text { Température de } \\
\text { pyrolyse en }{ }^{\circ} \mathbf{C}\end{array}$ & $\begin{array}{c}\text { Vitesse de chauffage } \\
\text { en }{ }^{\circ} \mathbf{C} / \mathbf{m i n}\end{array}$ & $\begin{array}{c}\text { Palier d'isotherme } \\
\text { en min }\end{array}$ \\
\hline $\mathbf{1 0}$ & 24 & 450 & 7,5 & 90 \\
$\mathbf{2 0}$ & 24 & 450 & 7,5 & 90 \\
30 & $\mathbf{2 4}$ & 450 & 7,5 & 90 \\
$\mathbf{4 0}$ & 24 & 450 & 7,5 & 90 \\
30 & $\mathbf{1 2}$ & 450 & 7,5 & 90 \\
30 & $\mathbf{1 8}$ & 450 & 7,5 & 90 \\
30 & 24 & $\mathbf{3 0 0}$ & 7,5 & 90 \\
30 & 24 & $\mathbf{4 0 0}$ & 7,5 & 90 \\
30 & 24 & $\mathbf{5 0 0}$ & 7,5 & 90 \\
30 & 24 & 450 & 7,5 & $\mathbf{6 0}$ \\
30 & 24 & 450 & 7,5 & $\mathbf{1 2 0}$ \\
30 & 24 & 450 & 7,5 & $\mathbf{1 8 0}$ \\
\hline
\end{tabular}




\section{RESULTATS}

Les résultats de l'analyse immédiate sont présentés au Tableau 2. Cette biomasse présente des taux d'humidité et de cendre faibles. Par contre, le pourcentage en matière volatile est élevé. Ces derniers ont un effet sur l'obtention du charbon actif que nous détaillerons dans la partie discussion. En effet, la composition et les propriétés physiques de la biomasse sont des paramètres qui influencent la conversion de la biomasse lignocellulosique en charbon actif (Ousmaila, 2015).

Pour le rendement massique de pyrolyse, l'indice d'iode et l'indice de bleu de méthylène, la variation de chaque paramètre d'élaboration les fait augmenter et/ou diminuer comme nous montrent les Figures 2, 3 et 4 .

En effet, les valeurs du rendement massique de pyrolyse varient de $32,89 \%$ à $44,7 \%$. Elles donnent un pic avec la concentration, augmente avec le temps d'imprégnation et diminue avec la température de pyrolyse et le temps de séjour dans le four.

La variation (évolution) de concentration et de la température de pyrolyse fait augmenter les valeurs d'indice d'iode et le temps d'imprégnation et l'exposition à la température le fait diminuer. L'indice d'iode varie de $837,57 \mathrm{mg} / \mathrm{g}$ à $989,86 \mathrm{mg} / \mathrm{g}$ pour les charbons actifs élaborés. Le charbon actif commercial (CAC) a donné $989,86 \mathrm{mg} / \mathrm{g}$.

Quant à l'indice de bleu de méthylène, ses valeurs croissent avec l'évolution de la concentration de l'agent activant et décroissent avec le temps d'imprégnation. L'évolution des deux autres paramètres (température de pyrolyse et palier d'isotherme) ne donne pas des variations progressives des valeurs de l'indice de bleu de méthylène. Globalement, l'indice de bleu de méthylène varie de 411,98 mg/g à 613,17 $\mathrm{mg} / \mathrm{g}$, contre $603,26 \mathrm{mg} / \mathrm{g}$ pour le charbon actif commercial.

Les fonctions à la surface du charbon actif sont représentées au Tableau 3 pour quelques charbons actifs élaborés et le charbon actif commercial.

Les Figures 5 et 6 , présentent les courbes de détermination de $\mathrm{pH}$ au point de charge nulle. Les valeurs obtenues varient de 4,62 à 5,01 pour les charbons actifs élaborés et elle est de 6,84 pour le charbon actif commercial.

L'étude de l'optimisation de l'élimination de chrome est présentée aux Figures $7,11,12$, et 13 . La cinétique (variation de temps de contact) montre que pour les 5 premières minutes, l'adsorption est rapide, qui devient relativement lente. Mais le temps d'équilibre est allé à $2 \mathrm{~h}$. Ceci permet une étude des modèles cinétique du type pseudo-premier ordre, pseudo-second ordre et la diffusion intra particulaire par modélisation des équations 10,11 et 12 respectivement.

$$
\begin{aligned}
& \frac{1}{Q_{t}}=\frac{1}{Q_{e}}+\frac{K_{1}}{Q_{e} \times t} \\
& \frac{t}{Q_{t}}=\frac{1}{K_{2} Q_{2}^{2}}+\frac{t}{Q_{2}} \\
& Q_{t}=K_{p} \times t^{1 / 2}+C
\end{aligned}
$$

La quantité du chrome adsorbée évolue avec l'augmentation de la masse de l'adsorbat et de la concentration initiale de la solution de chrome (Figures 11 et 13). Quant au pH de la solution, son évolution fait diminuer la quantité du chrome adsorbée (Figure 12).

Ainsi, avec les conditions optimales d'élimination du chrome obtenues $(\mathrm{Ci}=70$ $\mathrm{mg} / \mathrm{L}, \mathrm{t}=2 \mathrm{~h}, \mathrm{~m}=80 \mathrm{mg}, \mathrm{pH}=2$ ) pour l'échantillon noté $\mathrm{H}_{3} \mathrm{PO}_{4}-12 \mathrm{~h}$, le Tableau 4 donne les résultats pour quelques charbons actifs élaborés et le charbon actif commercial. 
Tableau 2 : Résultats de l'analyse immédiate.

\begin{tabular}{ccc}
\hline Taux d'humidité en \% & Taux de cendre en \% & Matière volatile en \% \\
\hline 2,92 & 6,66 & 96,14 \\
\hline
\end{tabular}

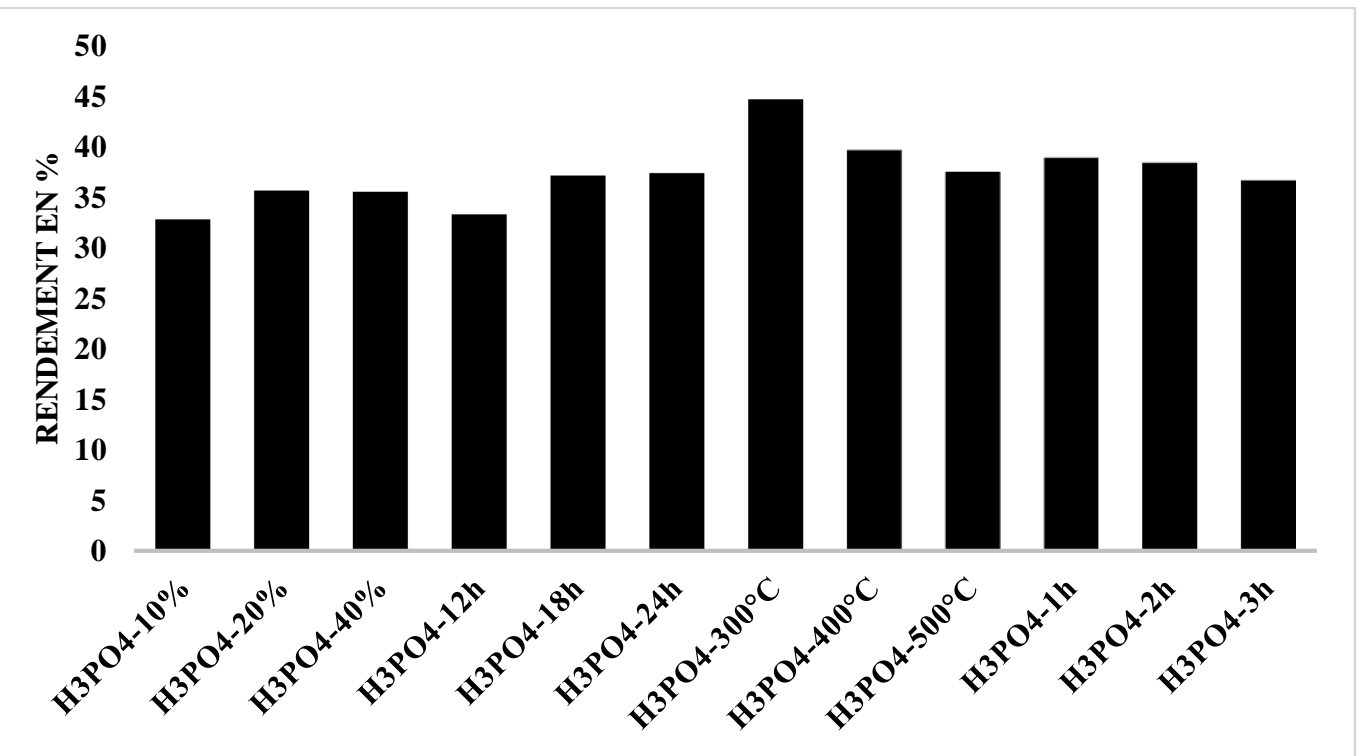

Figure 2 : Rendement massique de pyrolyse en $\%$.

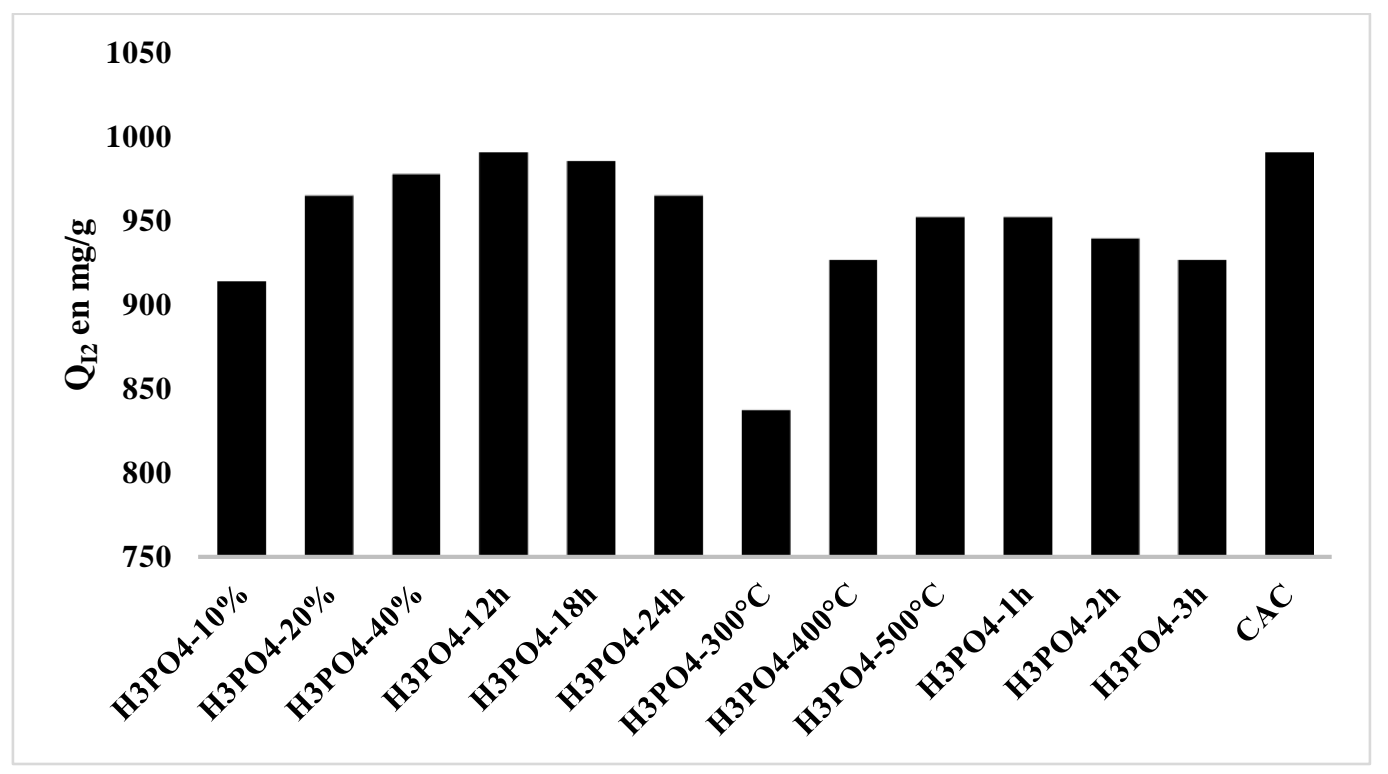

Figure 3 : Capacité d'absorption d'iode en mg/g. 


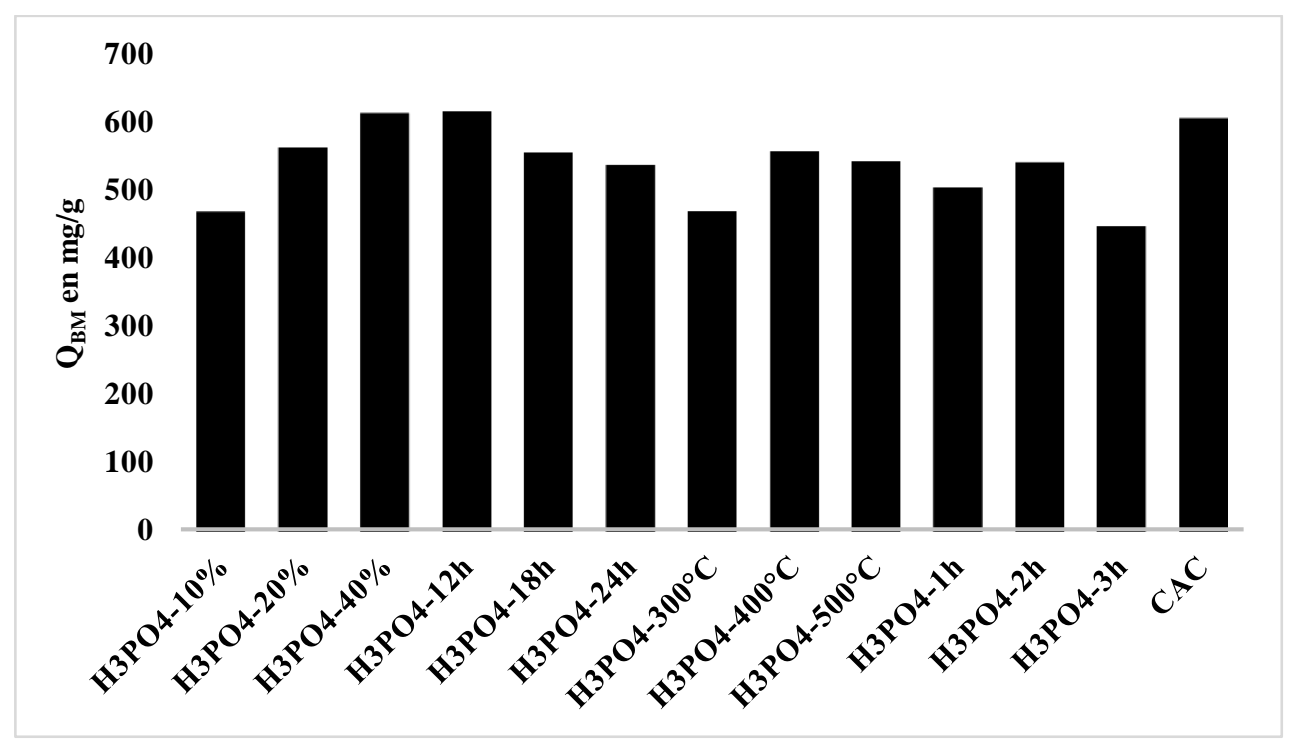

Figure 4 : Capacité d'adsorption de bleu de méthylène en mg/g.

Tableau 3 : Fonctions de surface de quelques échantillons en méq/g.

\begin{tabular}{ccccccc}
\hline $\begin{array}{c}\text { Charbons } \\
\text { actifs }\end{array}$ & carboxylique & lactone & phénol & carbonyle & Total acide & Totale basique \\
\hline H3PO4-12h & 0,8 & 3 & 0,6 & 1,4 & 5,8 & - \\
H3PO4-40\% & 1 & 1,6 & 0,7 & 2 & 5,3 & - \\
H3PO4-10\% & 0,5 & 2,3 & 0,4 & 1 & 4,2 & - \\
H3PO4-3h & 1,2 & 3,4 & 0,4 & 2,2 & 7,2 & - \\
CAC & - & 2 & 0,1 & 1,2 & 3,3 & - \\
\hline
\end{tabular}

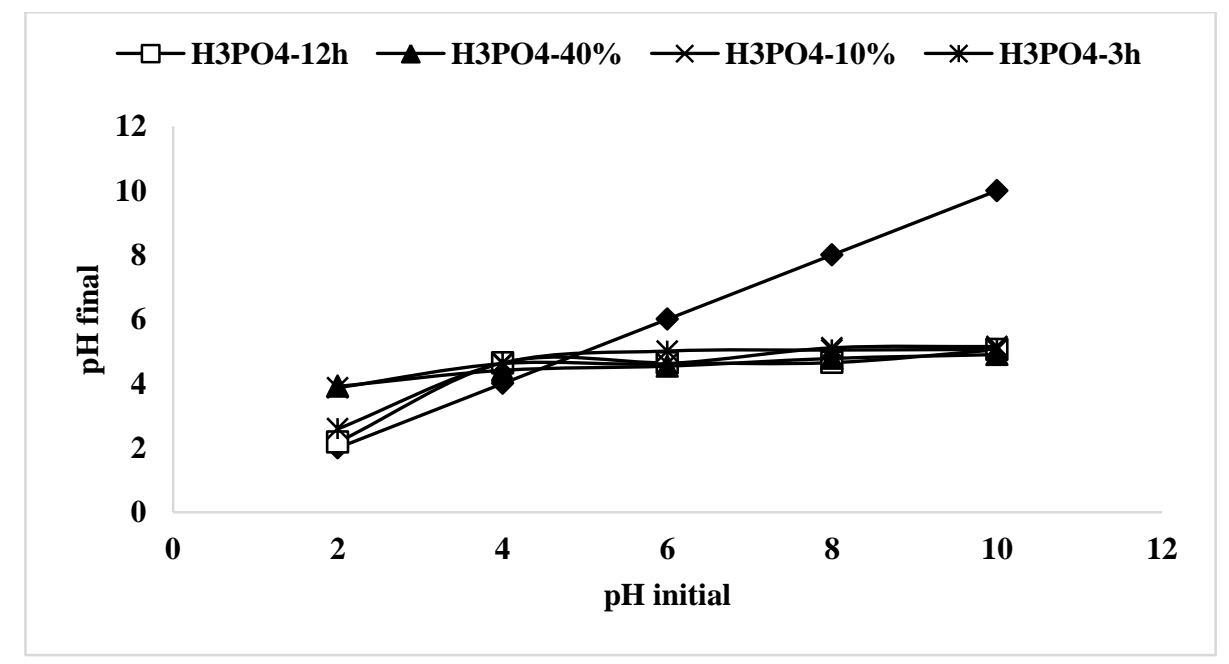

Figure 5 : Détermination de $\mathrm{pH}_{\mathrm{PCN}}$ de quelques charbons actifs élaborés. 


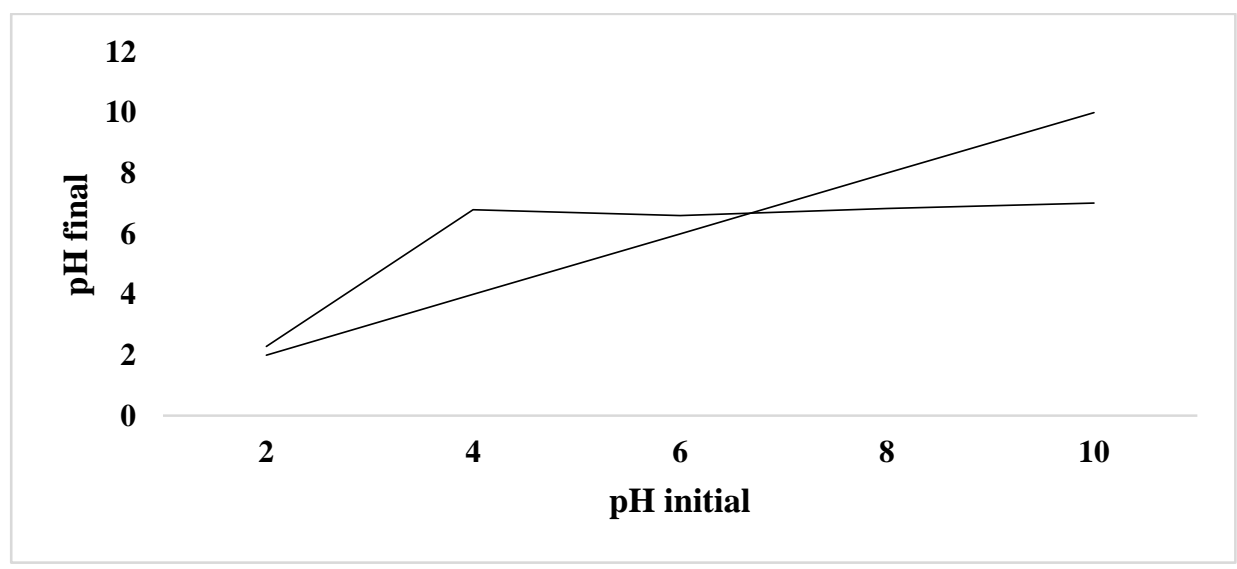

Figure 6 : Détermination de $\mathrm{pH}_{\mathrm{PCN}}$ de charbon actif commercial.

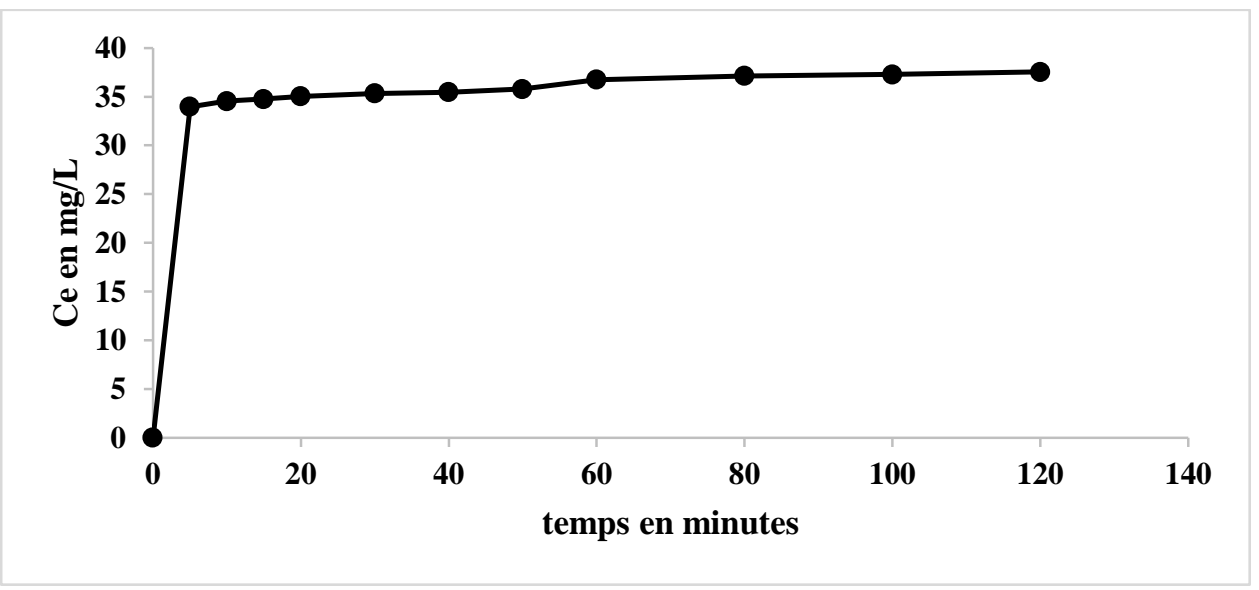

Figure 7 : Variation du temps de contact $(\mathrm{Ci}=50 \mathrm{mg} / \mathrm{L}, \mathrm{m}=50 \mathrm{mg})$.

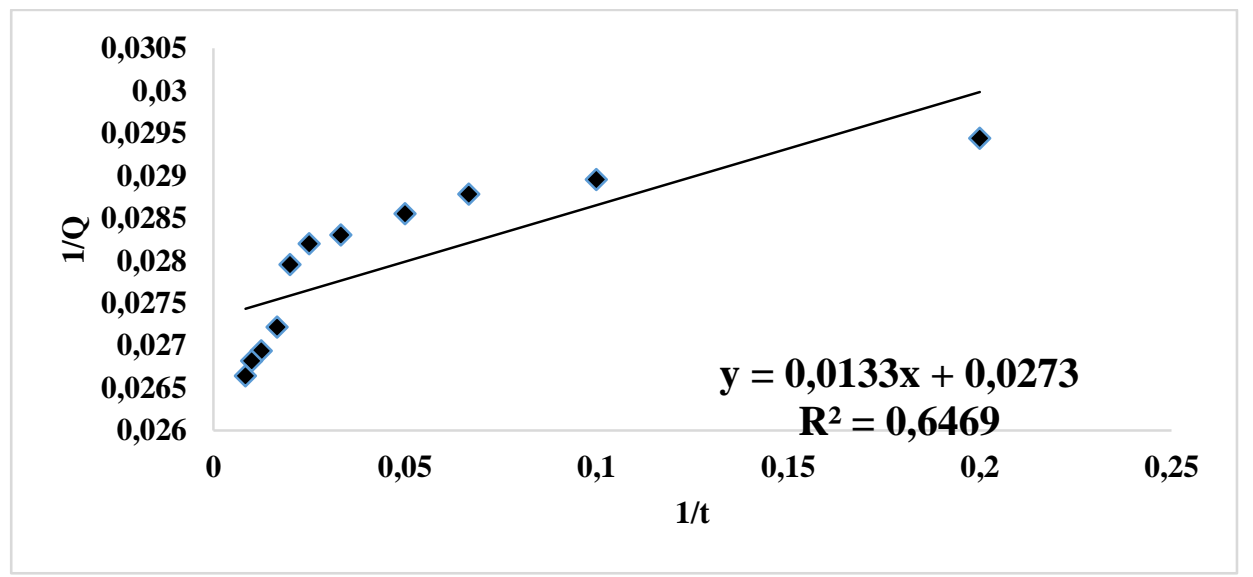

Figure 8 : Courbe de cinétique pseudo-premier ordre. 


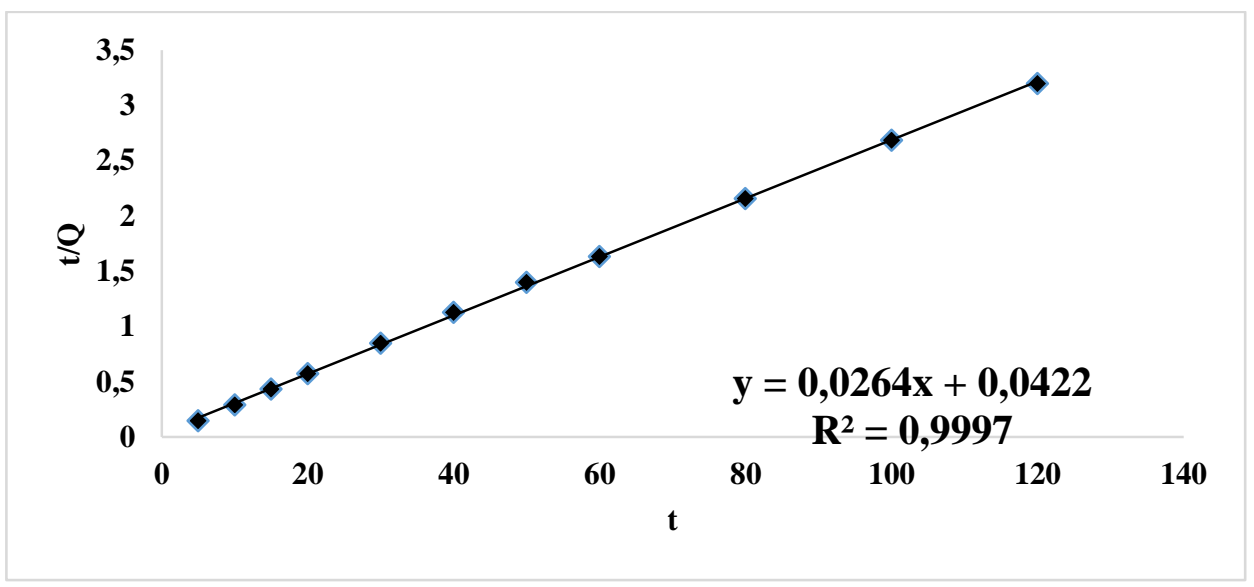

Figure 9 : Courbe de cinétique pseudo-second ordre.

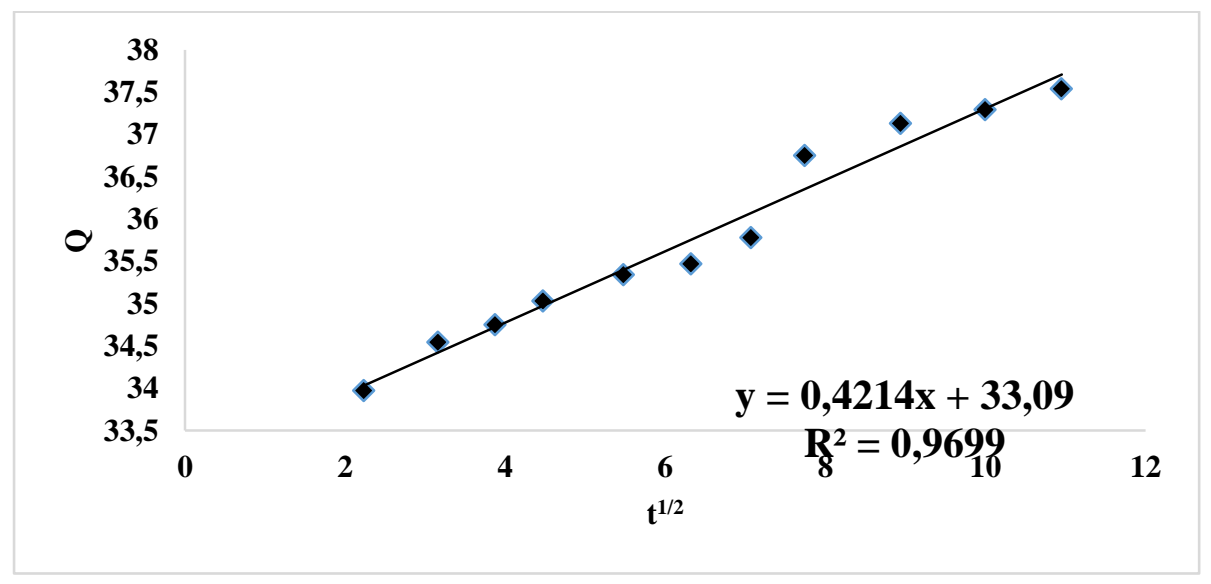

Figure 10 : Courbe de la diffusion intra particulaire.

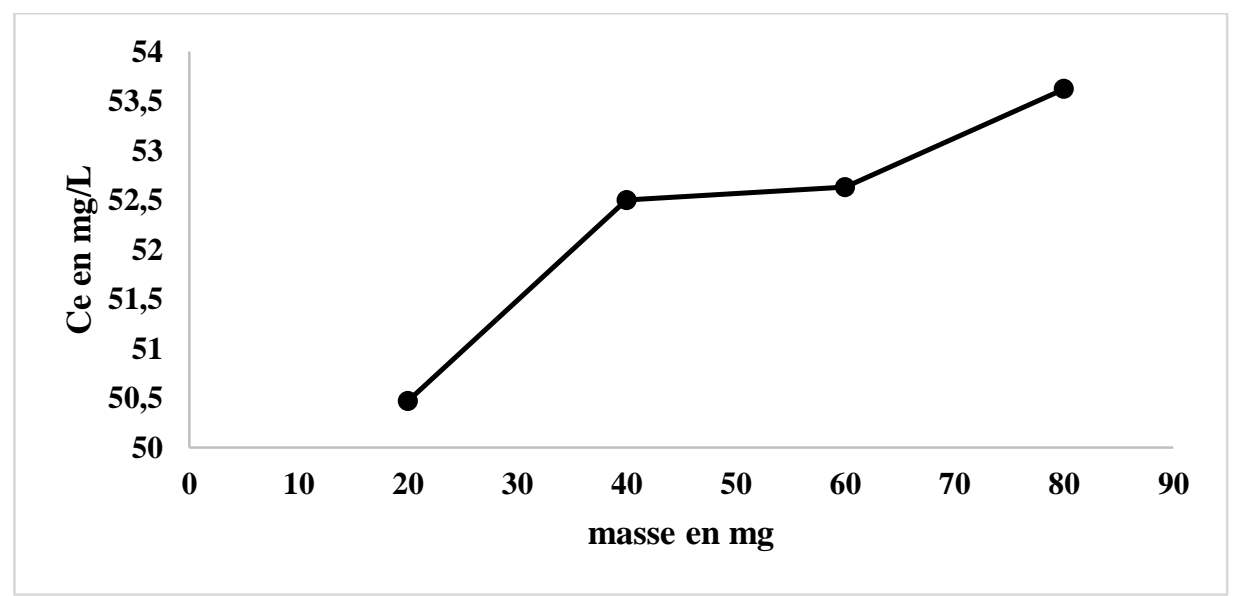

Figure 11 : Variation de la masse $(\mathrm{Ci}=70 \mathrm{mg} / \mathrm{L}, \mathrm{t}=2 \mathrm{~h})$. 


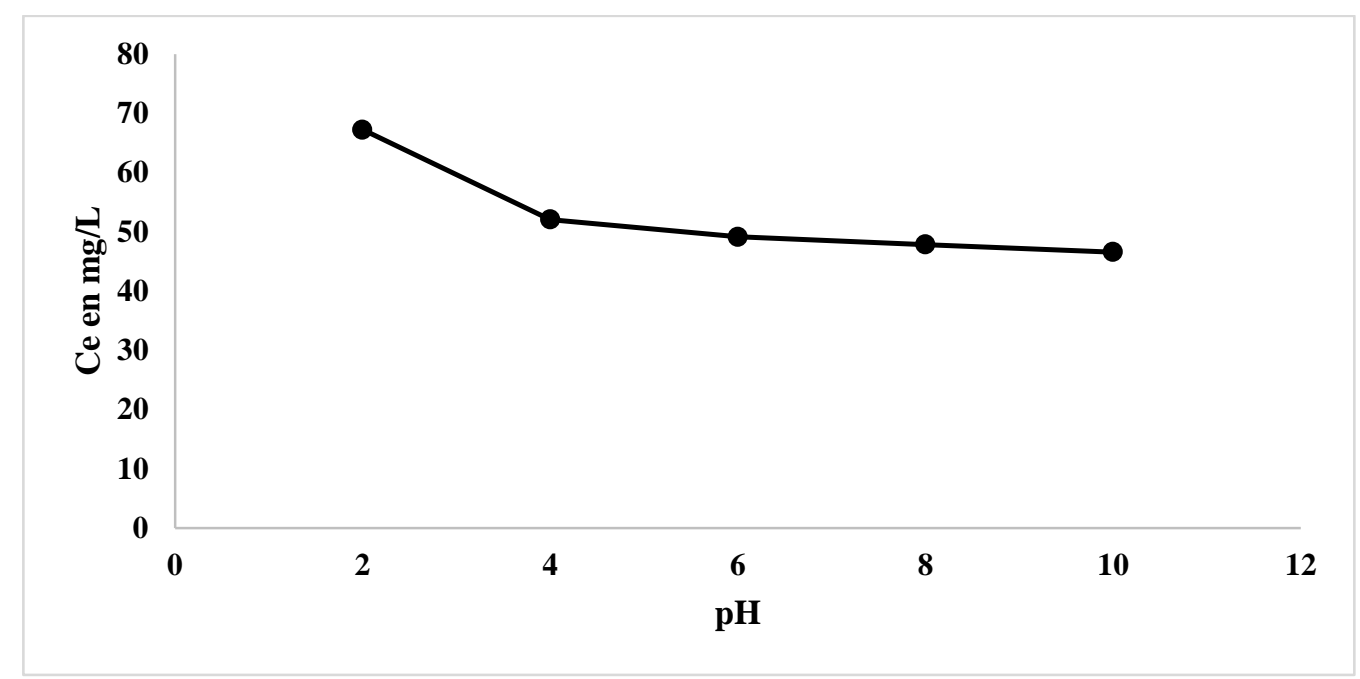

Figure 12 : Variation de $\mathrm{pH}(\mathrm{Ci}=70 \mathrm{mg} / \mathrm{L}, \mathrm{m}=80 \mathrm{mg}, \mathrm{t}=2 \mathrm{~h})$.

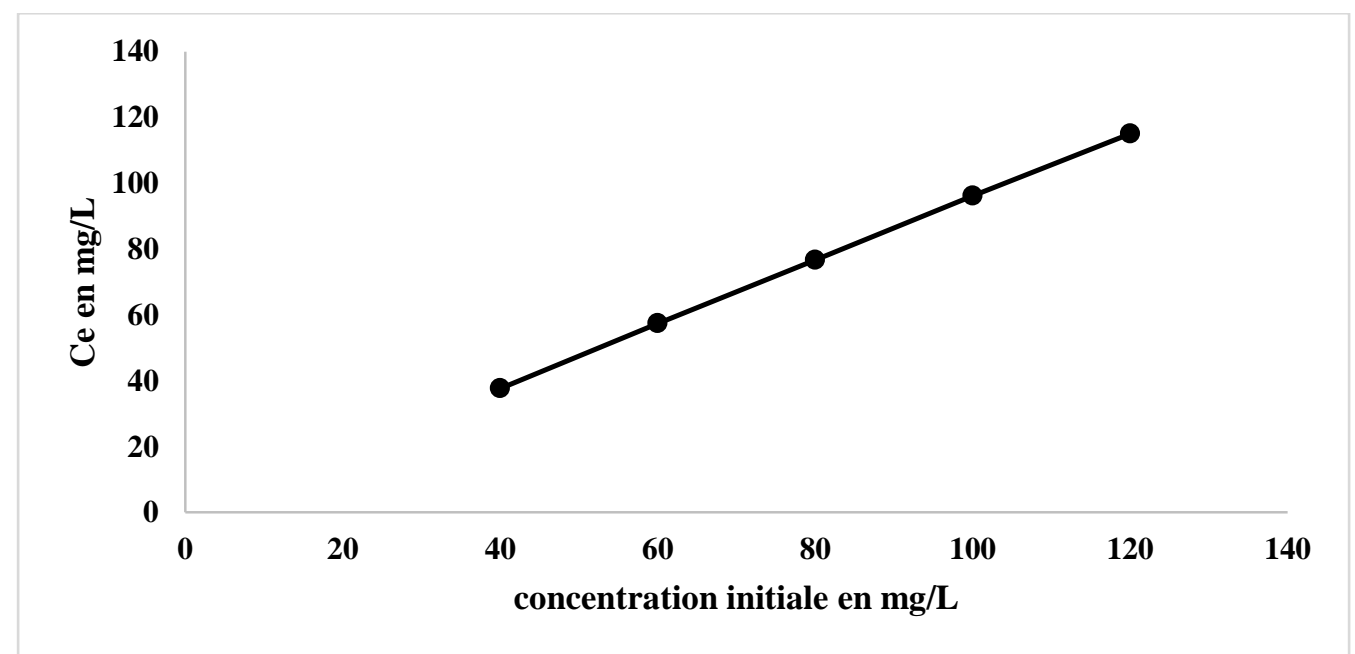

Figure 13 : Variation de la concentration initiale $(\mathrm{m}=80 \mathrm{mg}, \mathrm{pH}=2, \mathrm{t}=2 \mathrm{~h})$.

Tableau 4 : Résultats d'élimination du chrome VI $(\mathrm{Ci}=70 \mathrm{mg} / \mathrm{L}, \mathrm{m}=80 \mathrm{mg}, \mathrm{pH}=2, \mathrm{t}=2 \mathrm{~h})$.

\begin{tabular}{lccccc}
\hline & $\mathbf{C i}(\mathbf{m g} / \mathbf{L})$ & $\mathbf{C f}(\mathbf{m g} / \mathbf{L})$ & $\mathbf{C e}(\mathbf{m g} / \mathbf{L})$ & $\mathbf{Q}(\mathbf{m g} / \mathbf{g})$ & $\mathbf{R}(\boldsymbol{\%})$ \\
\hline H3PO4-12h & 70 & 2,76 & 67,24 & 42,025 & 96,057143 \\
H3PO4-40\% & 70 & 4,67 & 65,33 & 40,83125 & 93,328571 \\
H3PO4-10\% & 70 & 12,85 & 57,15 & 35,71875 & 81,642857 \\
H3PO4-3h & 70 & 0,86 & 69,14 & 43,2125 & 98,771429 \\
CAC & 70 & 5,96 & 64,04 & 40,025 & 91,485714 \\
\hline
\end{tabular}




\section{DISCUSSION}

Avec un taux d'humidité faible $(2,92 \%)$, le charbon actif élaboré pourrait avoir un haut pouvoir calorifique supérieur (PCS) (Mbaye, 2014). Le faible taux de cendre $(6,66 \%)$ implique que la biomasse est constituée essentiellement de la matière organique, donc de l'élément carbone. La haute teneur en matières volatiles $(96,14 \%)$ présente les avantages suivants pour l'élaboration du charbon actif : haut degré de graphitisation, haut PCS et haute quantité de groupes fonctionnels (Mbaye, 2014). Ces résultats laissent présager que la coque du noyau de balanites aegyptiaca est un bon précurseur pour l'élaboration du charbon actif.

La concentration en acide, le temps d'imprégnation, la température de pyrolyse ainsi que le temps de séjour dans le four sont des paramètres qui ont une influence sur le rendement massique. Cependant, l'augmentation de la concentration et le temps d'imprégnation ont pour effet d'augmenter le rendement massique. Ce qui est en accord avec les résultats trouvés par Ousmaila (2015) pour l'effet de la concentration d'acide ortho phosphorique. Ceci peut s'expliquer par le fait que $1^{\prime} \mathrm{H}_{3} \mathrm{PO}_{4}$ en tant que agent activant, est un agent déshydratant retardant la décomposition thermique, limitant les pertes de matières volatiles et menant à la formation d'une matrice carbonée rigide (Zhao et al., 2007). Par contre, une élévation de la température de pyrolyse ou de temps de séjour diminue le pourcentage en masse du charbon actif. Cette situation est un phénomène classique en thermochimie; sous l'effet d'une augmentation de la chaleur ou de la durée de son exposition, on assiste à plus de perte de macromolécules qui constituent la biomasse.

Les paramètres qui influent réellement sur l'indice d'iode sont la concentration de l'agent activant et la température de pyrolyse. La variation (élévation) de ces paramètres fait augmenter la capacité d'adsorption d'iode.
Plusieurs auteurs ont montré que cette dernière croit avec la concentration. C'est le cas de Ibrahim et al. (2012) pour l'activation des tourteaux de Karité et des tourteaux de coton. Cet indice varie de $837,57 \mathrm{mg} / \mathrm{g}$ à 989,86 mg/g. Ainsi, sur l'ensemble des CAEs, celui qui possède le meilleur indice d'iode est le $\mathrm{H}_{3} \mathrm{PO}_{4}-12 \mathrm{~h}$ avec $989,86 \mathrm{mg} / \mathrm{g}$. Cette valeur est la même obtenue avec le CAC. D'après la description technique de SILEX INTERNATIONAL sur le critère de qualité d'un charbon actif, l'indice d'iode doit être supérieur à $950 \mathrm{mg} / \mathrm{g}$. Parmi les charbons actifs élaborés, sept remplissent ce critère de performance.

Les variations des valeurs de l'indice de bleu de méthylène vont de 444,93 mg/g à $613,17 \mathrm{mg} / \mathrm{g}$. Ainsi, comme pour l'indice d'iode, la concentration de l'acide a un effet sur la porosité des charbons actifs. Aussi, la variation $\mathrm{du}$ temps d'imprégnation fait décroitre l'indice de bleu de méthylène de la meilleure valeur obtenue sur l'ensemble des échantillons à 534,17 mg/g. Pour les autres paramètres, l'effet de leur variation n'est pas linéaire. En effet, dans les conditions opératoires, deux de nos Charbons Actifs Elaborés (CAEs) ont développé des indices de bleu de méthylène plus grands $(613,17 \mathrm{mg} / \mathrm{g}$ et $610,61 \mathrm{mg} / \mathrm{g}$ ) que celui obtenu avec le CAC $(603,26 \mathrm{mg} / \mathrm{g})$.

Il est à noter que les échantillons ayant développés les meilleurs indices d'iode sont ceux qui ont les meilleurs indices de bleu de méthylène, en l'occurrence le $\mathrm{H}_{3} \mathrm{PO}_{4}-12 \mathrm{~h}$.

Les résultats présentés au Tableau 3 montrent que tous les charbons actifs ont un caractère acide. L'absence totale de fonction basique s'explique par le fait que les charbons actifs élaborés n'ont pas été mis en contact avec l'oxygène en dessous de $200{ }^{\circ} \mathrm{C}$ ou audessus de $700{ }^{\circ} \mathrm{C}$. Ils n'ont pas suivi non plus un traitement avec l'hydrogène et ils n'ont pas été dégazés à la température ambiante car 
c'est à cette étape qu'on introduit les fonctions basiques (Rabilou, 2015).

Les valeurs de $\mathrm{pH}$ au point de charge nulle des CAEs et commercial sont tous inférieurs à la neutralité $(\mathrm{pH}<7)$. Elles vont de 4,62 à 5,01 pour les charbons actifs élaborés. Le charbon actif commercial a donné 6,84. En effet, les valeurs obtenues pour les charbons actifs élaborés sont nettement différentes de celles trouvées par Rabilou (2015). Ceci s'explique par la méthode de lavage après l'élaboration. Pour le charbon actif commercial, la valeur trouvée n'est pas assez différente $(6,9)$.

Dans l'intervalle de temps de 5 à 120 minutes, la quantité de chrome adsorbée n'est que de $3,57 \mathrm{mg} / \mathrm{L}$ alors que pour les cinq premières minutes, elle était de $33,97 \mathrm{mg} / \mathrm{L}$ (Figure 6). Avec une concentration initiale de $50 \mathrm{mg} / \mathrm{L}$ et un temps de contact de $2 \mathrm{~h}$, le taux d'extraction est de 75,08\%. Ce qui est appréciable. Mais comme nous sommes en train d'optimiser l'extraction et pour éviter l'élimination totale avant le temps de contact choisit $(2 \mathrm{~h})$, par la suite, $\mathrm{Ci}$ sera considérée à $70 \mathrm{mg} / \mathrm{L}$. Cependant, pour déterminer le modèle cinétique de l'adsorption, les Figures 8,9 et 10 donnent les représentations des modèles cinétiques du type pseudo-premier ordre, pseudo-second ordre et la diffusion intra particulaire respectivement. La droite de régression de la Figure 9 donne le coefficient de corrélation le plus important $\left(\mathrm{R}^{2}=0,9997\right)$. Ce qui signifie que la cinétique d'adsorption du chrome hexavalent sur le charbon actif est du type pseudo-second ordre Anatole et al. (2012). Néanmoins, avec la diffusion intra particulaire ce coefficient est appréciable $\left(\mathrm{R}^{2}\right.$ $=0,9699)$. Ce qui laisse à dire que l'adsorption se fait avec diffusion Anatole et al. (2012). L'existence de cette diffusion intra particulaire dans la porosité interne du charbon est supportée par la présence de mésopores (Mbaye, 2014). La variation (augmentation) de la surface de contact donne une augmentation de la quantité de chrome adsorbée (Figure 11). La courbe traduisant cette variation n'est pas linéaire. C'est qui veut dire que la quantité adsorbée n'est pas proportionnelle à la masse de l'adsorbant. En effet la quantité adsorbée (concentration massique) et la masse sont deux grandeurs de nature différentes. La première est intensive et la seconde extensive. Néanmoins, le taux d'extraction passe de $72,1 \%$ à $76,6 \%$. Par contre, la capacité d'adsorption diminue avec l'augmentation de la masse et passe de $126,175 \mathrm{mg} / \mathrm{L}$ à $33,513 \mathrm{mg} / \mathrm{L}$. Ce qui est justifiable car la capacité d'adsorption est inversement proportionnelle à la masse. La courbe de la Figure 12 montre que l'adsorption du chrome par le CA est plus importante pour les valeurs de $\mathrm{pH}$ faibles. Ce qui a été confirmé par plusieurs auteurs dont Mbaye (2014), Frank Thierry (2012), Pal et Kaur (2013), .... Bien que ces derniers aient utilisé un autre adsorbant, l'adsorption reste toujours efficace à $\mathrm{pH}$ faible. En effet, les valeurs des $\mathrm{pH}$ au point de charge nulle des CAEs et du CAC sont tous supérieurs à 4 . Plus le $\mathrm{pH}$ d'une solution s'éloigne du $\mathrm{pH}_{\mathrm{PCN}}$ à valeurs inférieures, plus la charge globale du CA devient positive. Il aura donc plus d'affinité avec une espèce chargée négativement. Et c'est ce qui se passe avec l'espèce du chrome; $\mathrm{HCrO}_{4}{ }^{-}$. Quant à la variation de concentration, la courbe obtenue est un segment dont le prolongement donnera une droite qui passera par l'origine. Donc la capacité d'adsorption est proportionnelle à la concentration initiale de la solution. Néanmoins, les valeurs du rendement passent de $94,15 \%$ à $96,18 \%$. Cette légère variation montre que la concentration initiale n'a pas d'effet sur le rendement d'extraction dans les mêmes conditions.

Comparés aux résultats trouvés dans la littérature, les résultats du tableau 4 sont appréciables vue les conditions de travail. Le temps de contact est court $(2 \mathrm{~h})$ alors que 
d'autres vont jusqu'à 6 h. Cela a été compensé par la masse de l'absorbant (80 mg).

L'échantillon qui a donné le plus fort taux d'élimination est le $\mathrm{H}_{3} \mathrm{PO}_{4}-3$ h. Par contre il n'est pas celui qui possède des indices d'iode et de bleu de méthylène les plus élevés et que son $\mathrm{pH}_{\mathrm{PCN}}$ et la somme de ses fonctions de surface sont semblables à ceux d'autres échantillons. Il faut noter et que c'est l'échantillon dont l'écart entre les deux indices (indice d'iode et indice de bleu de méthylène) est plus important, et que c'est l'indice d'iode qui est le meilleur. Par conséquent, cet échantillon a une plus forte affinité vis-à-vis des molécules de taille relativement petite.

\section{Conclusion}

Le protocole mis au point pour l'activation de la coque de noyau de Balanites aegyptiaca a permis d'avoir des variétés de charbons actifs. Les résultats de la caractérisation ont monté que les charbons actifs élaborés sont dotés d'une porosité assez élevée.

Ainsi, les charbons actifs élaborés et le charbon actif commercial ont fait l'objet d'une application pour le traitement d'une solution chargée en chrome VI. Les essais d'élimination du chrome en solution aqueuse (solution de dichromate de potassium) ont donné des rendements d'extraction allant de $81,64 \%$ à $98,77 \%$ pour quelques charbons actifs élaborés et $91,48 \%$ pour le charbon actif commercial. Ces résultats ont été obtenus pour un temps d'équilibre de $2 \mathrm{~h}$, une masse en adsorbant (CA) de $80 \mathrm{mg}$, une concentration initiale de la solution de $70 \mathrm{mg} / \mathrm{L}$ ayant un $\mathrm{pH}$ $=2$.

\section{CONFLIT D'INTERETS}

Les auteurs déclarent qu'ils n'ont aucun conflit d'intérêts.

\section{CONTRIBUTIONS DES AUTEURS}

Dans ce document, les auteurs ont amené leur contribution comme suit: DBMS est l'investigateur principal; $\mathrm{HIH}$ a suivi et corrigé l'article ; MMMA et ZA ont participé à la correction de l'article ; IN a proposé le protocole expérimental.

\section{REFERENCES}

Anatole KKM, Philippe NV, Josaphat NDP, Daniel MWM, Gracien EB, Bernard IL, Joseph MM. 2012. Adsorption de la quinine bichlorhydrate sur un charbon actif peu coûteux à base de la Bagasse de canne à sucre imprégnée de l'acide phosphorique. Int. J. Biol. Chem. Sci., 6(3): 1337-1359. http://ajol.info/index.php/ijbcs

Clément KB, Moctar LB, Sèmiyou O, Martin A. 2015. Préparation des charbons actifs par voie chimique à l'acide phosphorique à base de coque de noix de coco. Int. $J$. Biol. Chem. Sci., 9(1): 563-580. http://ajol.info/index.php/ijbcs

Drissa B, Bini D, Albert T, Guessan Elogne Zor, Grah Patrick A, Didier R, Jean Victor W. 2009. Etudes comparées des méthodes de préparation du charbon actif, suivies d'un test de dépollution d'une eau contaminée au diuron. J. Soc Ouest-Afr. Chim., 028: 41- 52.

Elena FI. 2002. Etude de la carbonisation et l'activation de précurseurs végétaux durs et mous. Thèse de doctorat en science. Université de Neuchâtel, Neuchâtel, 146 $\mathrm{p}$

Frank Thierry K. 2012. Etude des performances des charbons actifs préparés à partir de biomasses tropicales pour l'élimination du chrome et diuron en milieu aqueux. Mémoire de master en environnement option assainissement. 2iE, Burkina Faso, 79 p.

Ibrahim T, Moctar BL, Djaneye-Boundjou G, Doni KS, Nambo P. 2012. Optimisation 
du procédé de préparation des charbons actifs par voie chimique $\left(\mathrm{H}_{3} \mathrm{PO}_{4}\right)$ à partir des tourteaux de Karité et des tourteaux de Coton. Int. J. Biol. Chem. Sci., 6(1): 461-478. http://ajol.info/index.php/ijbcs

Mbaye G. 2009. Synthèse et études des charbons actifs pour le traitement des eaux usées d'une tannerie. Mémoire de master en énergie et procédés industriels. 2iE, Burkina Faso, 52 p.

Mbaye G. 2014. Développement de charbon actif à partir de biomasse lignocellulosique pour des applications dans le traitement de l'eau. Thèse de doctorat en technologie de l'Eau, de l'Energie et de l'Environnement. 2iE, Burkina Faso, 215 p.

Ousmaila SM, Adamou Z, Ibrahim D, Ibrahim N. 2016. Préparation et caractérisation de charbons actifs à base de coques de noyaux de Balanites Eagyptiaca et de Zizyphus Mauritiana. J. Soc. Ouest-Afr. Chim., 041: 59-67.

Ousmaila SM. 2015. Elaboration des charbons actifs à partir de la coque de Balanites aegyptiaca et de la coque de Zizyphys mauritiana et application de ces derniers dans le traitement des solutions chargées en Iode et en Bleu de Méthylène. Mémoire de master en chimie inorganique. Niamey : UAM, 64p.
Pal J, Kaur M. 2013. Adsorption of hexavalent chromium from textile industry effluent by sawdust of Dalbergia Sisoo. Journal of Environmental Science and Sustainability (JESS), 1(1): 34-40. http://www.jessresearch.com.

Rabilou SM. 2015. Elaboration du charbon actif en poudre à partir de la coque de Balanites aegyptiaca et de la coque de Zizyphys mauritiaca. Mémoire de master en chimie inorganique. Niamey: UAM, $69 \mathrm{p}$.

Urbain Paul G, Koffi Marcellin Y, Brou Lazare Y, Aka Marcel K, Ardjouma D, Yao Urbain K, Kalédia Paul Honoré O, Donourou D, Albert T. 2015. Adsorption du benzo(a)pyrène sur du charbon activé à base de coques de coco provenant de Côte d'Ivoire. Int. J. Biol. Chem. Sci., 9(5): 2701-2711. http://ajol.info/index.php/ijbcs

Zhao J, Lai C, Dai Y, Xie J. 2007. Pore structure control of mesoporous carbon as supercapacitor material. Materials Letters, 61(23-24): 4639-4642. DOI: https://doi.org/10.1016/j.matlet.2007.02. 071. 